\title{
Three Markers in Cancerous and Healthy Cells of Patients with Non-Small-Cell Lung Carcinoma (NSCLC)
}

\author{
Hamidreza Jamaati', Adnan Khosravi², Atefeh Abedini ${ }^{3}$, Arda Kiani ${ }^{3}$, Payam \\ Tabarsi $^{4}$, Hossein Dargahi ${ }^{5}$, Naghmeh Bahrami ${ }^{6,7}$, Mehdi Kazempour Dizaji ${ }^{8}$, \\ Farahnaz Sadegh Beigee', Mihan Pourabdolah ${ }^{1}$, Abbas Gheysouri ${ }^{1}$, Nasser \\ Ahmadian ${ }^{10}$, Masoum Abniki ${ }^{11}$, Abdolreza Mohamadnia ${ }^{1,12 *}$, Ali Akbar Velayati ${ }^{13}$
}

\begin{abstract}
Introduction: Lung cancer is the most common cause of cancer-related death among males and females. The diagnosis of lung cancer is of great importance for clinical considerations and follow-up treatment. This study aimed to examine the expression of CEA, LUNX, and CK19 biomarkers in the cancerous and healthy tissues of patients suffering from NSCLC. Methods: In this study, 30 patients with NSCLCs referring to Masih Daneshvari Hospital in Tehran were voluntarily selected prior to taking any treatment. A tissue sample from the center and a sample of healthy tissues close to the cancerous masses were prepared by a specialist in the bronchoscopy sector and tested using real-time RT-PCR. Results: Positive CEA $m R N A$ was observed in cancerous tissues in the center of tumors of 25 out of 30 cases. In the healthy tissue group, the same was found in 10 out of 30 cases $(\mathrm{P}<0.001)$. The markers $C K 19$ and $L U N X$ mRNAs showed to be positive in cancerous samples in the center of tumors of 15 and 22 out of 30 cases, and in the healthy tissue group, the expression was observed in 5 and 4 out of 30 cases, respectively $(\mathrm{P}<0.001)$. Conclusion: This study confirms that the aformentioed markers are the ones with a relatively appropriate sensitivity and specificity for the diagnosis of lung cancer.
\end{abstract}

Keywords: Lung cancer- biomarker- LUNX-CK19-CEA

Asian Pac J Cancer Prev, 20 (8), 2281-2285

\section{Introduction}

Lung cancer is the most common cause of cancer death among men and women, and its prevalence appears to be lower in some races such as white and African-American men. The main reason of lung cancer is smoking, and any other tools like pipe or hookah lead to similar conditions as a cigarette (Thress et al., 2015). The chemicals in cigarettes are hazardous to those who are indirectly involved in and exposed to smoking and mostly result in lung cancer. In the United States, the second cause of lung cancer has been reported to be radon gas (Rosell et al., 2009).

The behavior of lung tumors also depends on the type of pathology. Lung cancer is known as lung small cell lung cancer (SCLC) and non-small cell lung cancer (NSCLC).

The epithelial lung cancers are categorized into two primary types, including small-cell lung carcinoma (SCLC) and non-small-cell lung carcinoma (NSCLC). The latter group is subclassified into adenocarcinoma, squamous cell carcinoma, and large cell carcinoma. All these four types account for $90 \%$ of epithelial lung cancers (Lei et al., 2013).

A biomarker is a biochemical molecule found in blood or other body fluids or tissues and is indicative of a normal or an abnormal process and a specific condition or disease (Grunnet and Sorensen, 2012). Nowadays, a challenge in the diagnosis of cancer is to find a close relationship between the biomarkers and the clinical symptoms of the disease and also to have a non-aggressive diagnostic method in the early stages of the disease (Bhatt et al., 2010).

Diagnostic and prognostic biomarkers are cancer

\footnotetext{
${ }^{1}$ Chronic Respiratory Diseases Research Center, ${ }^{2}$ Tobacco Prevention and Control Research Center, ${ }^{3}$ Tracheal Diseases Research Center, ${ }^{4}$ Clinical Tuberculosis and Epidemiology Research Center, ${ }^{8}$ Biostatistics Department, Mycobacteriology Research Center, Masih Daneshvari Hospital, ${ }^{9}$ Lung Transplantation Research Center, ${ }^{13}$ Mycobacteriology Research Centre (MRC), National Research Institute of Tuberculosis and Lung Diseases (NRITLD), ${ }^{12}$ School of Advanced Technologies in Medicine, Shahid Beheshti University of Medical Sciences, ${ }^{5}$ Allied Medicine, Health information Management Research Center, ${ }^{6}$ Craniomaxillofacial Research Center, ${ }^{77}$ Department of Tissue Engineering and Applied Cell Sciences, School of Advanced Technologies in Medicine, Tehran university of Medical Sciences, ${ }^{10}$ Transplantation Center, Department of Curative Affairs, Ministry of Health and Medical Education, ${ }^{11}$ Department of Biotechnology, Faculty of Sciences, Islamic Azad University, Tehran, Iran. *For Correspondence: mohamadnia.ar@gmail.com
} 
screening tests that assist oncologists in the diagnosis of the disease at an early stage in patients with cancer; specifically, those with a higher risk of disease. Biomarkers are also used to screen the disease response to the treatment (Benedíková et al., 2012).

$C E A$ (carcinoembryonic antigen) is a cell surface glycoprotein and a known tumor marker for lung cancer. CEA plays a role in cell adhesion; it is normally synthesized during the development of embryo, and its synthesis stops shortly before birth (Grunnet and Sorensen, 2012). The increased $C E A$ level provides prognostic information on the disease process.

LUNX (lung-specific X protein) is a lung-specific gene, which is highly expressed in the NSCLC. According to a large number of evidence, the $L U N X$ may be a diagnostic biomarker for lung cancer (Iwao et al., 2001; Wallace et al., 2005). Sequential analysis has indicated that LUNX may have a role in inherent safety (Mitas et al., 2003).

CK19 (cytokeratin 19) belongs to a family of keratins, and its major role function is to maintain the integrity of the epithelial cell. This protein also has a critical role in the cell cycle of stress-response and apoptosis. CK19 is an extremely useful marker for the tumor diagnosis and control (Zaghloul, 2015) .

$18 s r R N A$ (18s subunit of ribosomal RNA) has widely been discussed as a house-keeping gene in similar studies and was used as a reference gene in the current study (Sardareh et al., 2014).

The main objective of this study was to determine the LUNX mRNA,CEA $m R N A$, and CK19 mRNA in the NSCLC tissue samples using the real-time PCR method and to examine the sensitivity and specificity of these markers in the patients suffering from NSCLC. In future, they could help to diagnose the disease at the early stage prior to metastases becoming apparent.

\section{Materials and Methods}

\section{Method}

In this study, 30 patients with NSCLC (with the mean age of 49 and standard deviation of 10.4) referring to Masih Daneshvari Hospital in Tehran were voluntarily selected prior to taking any treatment. After obtaining ethical code No. IR.SBMU.NRITLD.REC.1396.343., two tissue samples were prepared by a specialist in the Bronchoscopy Sector of ..... and transported to the laboratory by a nitrogen tank. The first sample was procured from the center and the second one from healthy tissues close to the cancereous masses.

\section{RNA extraction}

For RNA extraction, RNA was first isolated from the samples by using the Cinna pure RNA kit (Cat no. PR891620-S, Sinaclon, Iran). Subsequently, a 20-25 $\mathrm{mg}$ piece of fresh tissue was prepared and grinded. The grinded tissue was mixed and homogenized with $400 \mu \mathrm{l}$ of the lysis solution. The precipitation solution was then added to the homogenized mixture, and the resulting solution was transmitted to the extraction column and centrifuged.
In the next step, wash buffers I and II were used and poured on the column, respectively, and then both buffers were centrifuged. Finally, $50 \mathrm{~nm} \mu \mathrm{l}$ of RNase-free water was added to the column, and the pure RNA in the column was extracted. The RNA quality was finally evaluated by a Nano-droplet device.

\section{Complementary DNA (cDNA) preparation}

To prepare the cDNA from the extracted mRNA, Viva 2-steps Reverese transcriptase kit (Vivantis, Cat no. RTPL12, Malaysia) was applied. As per manufacturer's instructions, this kit has high sensitivity and specificity to reproduce any type of DNA from the RNA pattern. The specific primers of each marker were designed with the aid of AlleleID7 software and ordered to be prepared. Table 1 lists the parameters of the primers and their rates in the final real-time reaction.

\section{Real-time quantitative reverse transcription (RT)-PCR}

Real-time RT-PCR was performed with GeneAmp 5,700 sequence detection system (Foster City, CA, USA). The real-time RT-PCR reaction components were composed of (A) $2 \mu \mathrm{L}$ of the template, (B) $4 \mu \mathrm{L}$ of the Master mix, (C) primer with optimal concentration found in set up tests, and (D) deionized distilled water to reach a final reaction volume of $20 \mu \mathrm{L}$. Positive and negative controls were also used simultaneously for quality control and the detection of possible contamination. Initial step of RT-PCR was conducted at $50^{\circ} \mathrm{C}$ for 2 minutes, followed by a 10 -minute hold at $95^{\circ} \mathrm{C}$. Cycles ( $\mathrm{n}=40$ first round) consisted of a 15 -second melt at $95^{\circ} \mathrm{C}$, accompanied by a 1 -minute annealing/extension at $60^{\circ} \mathrm{C}$. The final step was performed at $60^{\circ} \mathrm{C}$ incubation for 1 minute. All reactions were performed in triplicate, and a negative control lacking cDNA was included.

Relative differences between the expression of $C E A$ $m R N A, C K 19 m R N A$, and $L U N X m R N A$ were measured for both the patients and healthy participants using the $\Delta \Delta \mathrm{Ct}$ method.

\section{Statistical analysis}

The sample size was estimated based on the positive markers in the two groups with considering 5\% and 20\% type I and type II errors.

Comparison of the mean of the two groups was done by t-test and comparing the markers positivity in the two groups using the two-sample binomial test using SPSS21 software. The statistical difference was considered significant at $\mathrm{P} \leq 0.05$.

\section{Results}

The present work was performed using a tissue sample of 30 patients, of whom 8 were female and 32 were male. The participants' mean age was 49 with a standard deviation of 10.4. There was no significant difference between the mean of samples obtained at the center and sides of the tumor $(\mathrm{P}=0.058)$. Accordingly, the $18 s R N A$ gene was selected as a reference gene and included in the study. 
Table 1. Specifications of Primers Used in Real-Time RT-PCR Reaction

\begin{tabular}{lcccc}
\hline Parameters & CEA & CK19 & LUNX & Mss rRNA \\
\hline Accessing number & M29540 & NM_002276.4 & M29540 & 002276.4 \\
Primer F & ACCCTGGATGTCCTCTATGG & TCCGAACCAAGTTTGAGAC & CCACCGTCTCTATGTCACCA & GTAACCCGTTGAACCCCATT \\
Primer length & 20 & 19 & 20 & 20 \\
Primer R & CAGGCATAGGTCCCGTTATTA & AATCCACCTCCACACTGA & GCCAAGTCCATCAAGCAGA & CCATCCAATCGGTAGTAGCG \\
Primer length & 21 & 18 & 19 & 20 \\
Product length & 174 & 222 & 211 & 152 \\
\hline
\end{tabular}

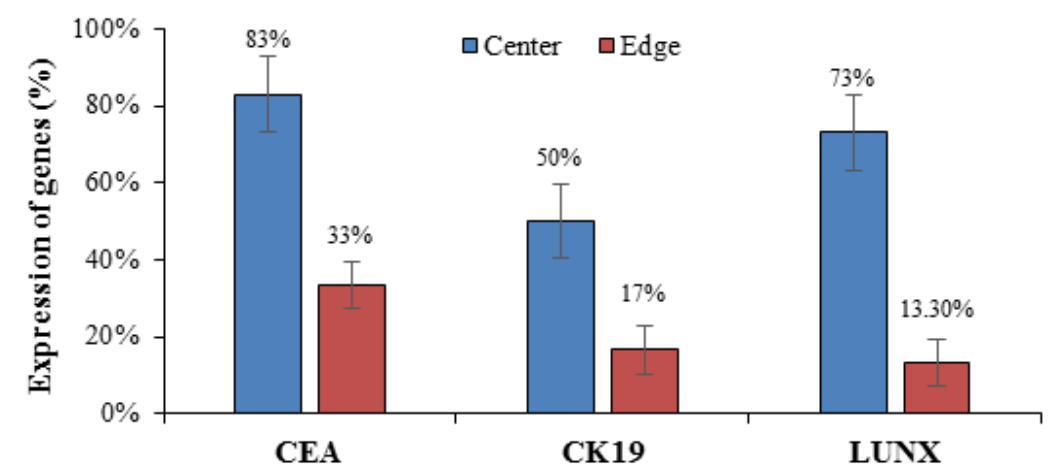

Figure 1. Expression of CK19, LUNX, and CEA mRNAs in the Center of Tumor and Its Side Tissues.

\section{Expression analysis of studied markers}

Following the extraction of the real-time RT-PCR reaction, the patients in each of the two groups who showed positive results for the expression of markers were determined.

The results showed the positive expression of the $C E A$ $m R N A$. Such a positive expression was also observed for cancerous center tissue samples in 25 of 30 cases; therefore, the sensitivity of this marker was determined to be $83 \%$. In the healthy side tissue group, the expression was found in 10 out of 30 cases. The comparative analysis of the gene expression between the two groups by two-sample binomial test represented a significant difference $(\mathrm{P}<0.001)$.

The expression of CK19 and LUNX mRNAs were conducted in 15 and 22 out of 30 patients, respectively. The sensitivity of the two markers was determined to be $50 \%$ and $73 \%$, respectively. However, in the healthy side tissue group, CK19 and LUNX mRNAs were detected in 5 and 4 out of 30 samples, respectively. The comparison of the gene expression, using two-sample binomial test, demonstrated a significant difference between the two groups of samples $(\mathrm{P}<0.001$; Figure 1).

Differences between the expression of markers in two sample groups

The $\Delta \Delta \mathrm{Ct}$ values for the $C E A, C K 19$, and $L U N X$ $m R N A s$ were calculated to be $-1.4,-1.2$, and -0.8 , respectively, which mathematically indicates that the number of initial versions for the markers in patients is $2.64,2.30$, and 1.74 times as much as that of healthy subjects, respectively (Figure 2).

\section{Discussion}

Lung cancer remains the most leading reason of cancer-related death among men and women. Smoking has been reported to be the main cause of lung cancer in the world (Thress et al., 2015), but in the United States, the second cause of lung cancer seems to be radon gas. Exposure to the radon gas along with smoking a cigarette significantly increases the likelihood of lung cancer (Rosell et al., 2009).

In this study, three biomarkers LUNX, CK19, CEA

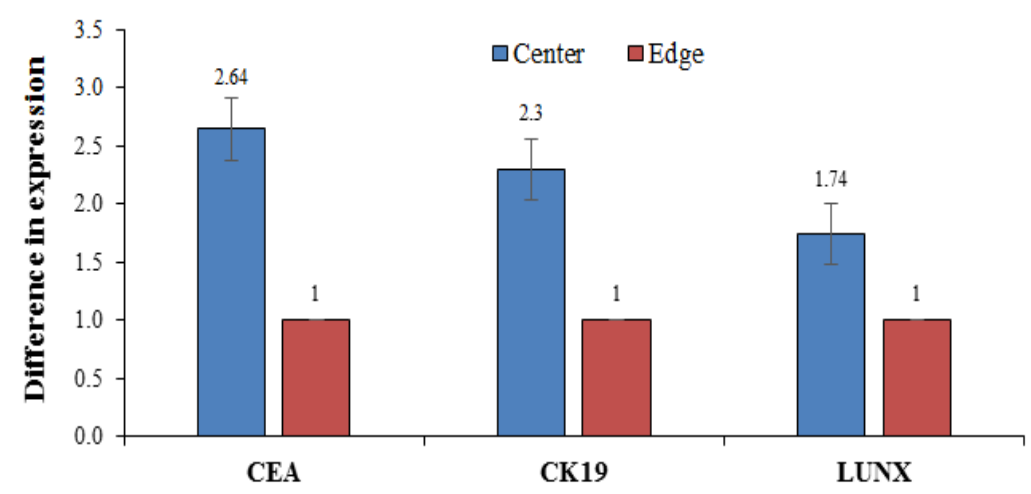

Figure 2. Difference in Expression of CEA, CK19, and LUNX $m R N A s$ in the Center of Tumor and Its Side Tissues 
were studied. It was shown that the expression of these biomarkers in cancerous tissue is significantly higher than healthy tissue. These results can help in the diagnosis and treatment.

Lung cancer mainly reveals no symptoms in the early stages; however, they emerge with the spread of cancer. The most frequent symptoms include respiratory problems such as cough, bloody sputum, systemic body complications like weight loss, etc (Marchetti et al., 2005).

Tumor markers are biochemical molecules of protein or glycoprotein generated in response to the emergence of cancer and can be detected in the blood, urine, and tumor tissues (Duffy, 2001; Thomas and Sweep, 2001). RNA markers can serve as the diagnostic tests, an advantage that persuaded the researcher to include them in the current study (Ghossein and Bhattacharya, 2000). Another advantage is detecting the small amounts of these markers by the use of the real-time RT-PCR, which is a high-sensitive method with great features. However, other protein markers, which are mainly in extremely small amounts, cannot be measured .

In a previous study, the expression of $C K 19$ and $C E A$ $m R N A s$ was investigated in the peripheral blood of NSLC patients. The rate of positive expression was indicated to be $57 \%$ for CK19 $m R N A$ and $40 \%$ for CEA $m R N A$ and $43 \%$ for both, suggesting that both mRNAs are suitable markers for the diagnosis of the micro-metastases (Zhu et al., 2004).

A number of studies have reported high embryonic carcinoma antigen in the serum levels of patients with NSCLC, as compared to those suffering from other types of cancer (Pastor et al., 1997; Oyama et al., 2003). This finding is in the same line with the findings of the present study. Wang et al., (2008) examined CK19 and LUNX $m R N A s$ expression on tissue and lymph node samples of 90 NSCLC patients. The expression of each marker showed to be significantly higher in patients with NSCLC than those with benign lung cancers (Wang et al., 2008), which is in good agreement with our results. Likewise, Yu et al., (2014) evaluated the expression of LUNX, CEA, and $C K 19$ mRNAs in 113 patients with lung cancer (case group) and 30 healthy subjects (control group). They found that the positive expression of $L U N X m R N A$ in peripheral blood and CEA and CK19 mRNAs in serum was significantly higher in the case group relative to the control group (Yu et al., 2014). In a study by Karimi et al., (2015) on the expression of CEA mRNA and LUNX $m R N A$ in 30 NSLC patients, there were 24 cases with positive expression of CEA $m R N A s$, indicating $80 \%$ sensitivity, and 21 cases with positive expression of $L U N X$ $m R N A$, suggesting the sensitivity of about $70 \%$. These two biomarkers were suggested as two markers with an appropriate sensitivity and specificity for lung cancer detection, which supports the results of the present study.

It should be noted that there were limitations in doing this research, such as access to more numbers and limited resource use. However, good results were achieved.

In conclusion, to conclude, the result of this pilot study on tumor markers of lung cancer can be applied to introduce a diagnostic test for lung cancer disease. To proof this finding, further studies with a larger sample size are recommended.

\section{Acknowledgements}

This research was extracted from a project during 2016-2017 in Massih Daneshvari Hospital, Shahid Beheshti University of Medical Sciences, Tehran, Iran. The author would like to express his sincere gratitude to the professors and colleagues in Masih Daneshvari Hospital.

\section{References}

Benedíková A, Srovnal J, Szkorupa M, et al (2012). Biomarkers in the detection of minimal systemic dissemination in lung cancer patients. Rozhl Chir, 91, 209-15.

Bhatt AN, Mathur R, Farooque A, et al (2010). Cancer biomarkers-Current perspectives. Indian J Med Res, 132, 129-49.

Duffy M (2001). Clinical uses of tumor markers: a critical review. Crit Rev Clin Lab Sci, 38, 225-62.

Ghossein R, Bhattacharya S (2000). Molecular detection and characterisation of circulating tumour cells and micrometastases in solid tumours. Eur J Cancer, 36, 1681-94.

Grunnet M, Sorensen J (2012). Carcinoembryonic antigen (CEA) as tumor marker in lung cancer. Lung Cancer, 76, 138-43.

Iwao K, Watanabe T, Fujiwara Y, et al (2001). Isolation of a novel human lung-specific gene, LUNX, a potential molecular marker for detection of micrometastasis in non-small-cell lung cancer. Int J Cancer, 91, 433-7.

Lei L, Huang Y, Gong W (2013). miR-205 promotes the growth, metastasis and chemoresistance of NSCLC cells by targeting PTEN. Oncol Rep, 30, 2897-902.

Marchetti A, Martella C, Felicioni L, et al (2005). EGFR mutations in non-small-cell lung cancer: analysis of a large series of cases and development of a rapid and sensitive method for diagnostic screening with potential implications on pharmacologic treatment. J Clin Oncol, 23, 857-65.

Mitas M, Hoover L, Silvestri G, et al (2003). Lunx is a superior molecular marker for detection of non-small lung cell cancer in peripheral blood. J Mol Diagn, 5, 237-42.

Oyama T, Kawamoto T, Matsuno K, et al (2003). A case-case study comparing the usefulness of serum trace elements $(\mathrm{Cu}$, $\mathrm{Zn}$ and Se) and tumor markers (CEA, SCC and SLX) in nonsmall cell lung cancer patients. Anticancer Res, 23, 605-12.

Pastor A, Menendez R, Cremades MJ, et al (1997). Diagnostic value of SCC, CEA and CYFRA 21.1 in lung cancer: a Bayesian analysis. Eur Respir J, 10, 603-9.

Rosell R, Moran T, Queralt C, et al (2009). Screening for epidermal growth factor receptor mutations in lung cancer. N Engl J Med, 361, 958-67.

Sardareh HM, Goodarzi MT, Yadegar-Azari R, et al (2014). Prostate cancer antigen 3 gene expression in peripheral blood and urine sediments from prostate cancer and benign prostatic hyperplasia patients versus healthy individuals. Urol J, 11, 1952-8.

Thomas C, Sweep C (2001). Serum tumor markers: past, state of the art, and future. Int J Biol Markers, 16, 73-86.

Thress KS, Paweletz CP, Felip E, et al (2015). Acquired EGFR C797S mutation mediates resistance to AZD9291 in non-small cell lung cancer harboring EGFR T790M. Nat Med, 21, 560-2.

Wallace MB, Block MI, Gillanders W, et al (2005). Accurate molecular detection of non-small cell lung cancer metastases 
in mediastinal lymph nodes sampled by endoscopic ultrasound-guided needle aspiration. Chest, 127, 430-7.

Wang W, Cui Y, Yao S (2008). Message RNA expression of $L U N X$, CK19 and CEA genes in NSCLC with micrometastasis in lymph nodes. CJO, 30, 121-4.

Yu H, Huang X, Zhu Z, et al (2014). Significance of combined detection of LunX mRNA and tumor markers in diagnosis of lung carcinoma. Chinese J Cancer Res, 26, 89.

Zaghloul M (2015). Cytokeratin 19 (CK19) as a Tumor Marker in Pleural Effusion. Trop Med Surg 3: e122. doi: 10.4172/23299088.1000 e122 Page 2 of 2 blood of patients with operable breast cancer: evaluation of their prognosti c significance. J Clin Oncol, 20, 3404-12.

Zhu G, Liu D, Wang X, et al (2004). Detection of CK19 and CEA mRNA expression for the diagnosis of peripheral blood micrometastases in patients with non-small cell lung cancer. CJLC, 7, 226-9.

\section{cc) (i) (9)}

This work is licensed under a Creative Commons AttributionNon Commercial 4.0 International License. 